\title{
Diagnosis of endometriotic lesions by sonovaginography with ultrasound gel
}

\author{
Elvira Brătilă ${ }^{1,2}$, Diana-Elena Comandaşu ${ }^{1,2}$, Ciprian Coroleucăă ${ }^{1,2}$, Monica Mihaela Cîrstoiu ${ }^{1,3}$, \\ Costin Berceanu ${ }^{4}$, Claudia Mehedintu1,5, Petre Bratila ${ }^{1,6}$, Simona Vladareanu ${ }^{1,7}$
}

1"Carol Davila" University of Medicine and Pharmacy, Bucharest, "'St. Pantelimon" Emergency Clinical Hospital, Bucharest, ${ }^{3}$ Emergency Universitary Hospital, Bucharest, ${ }^{4}$ University of Medicine and Pharmacy, Craiova, ${ }^{5 \text { "Nicolae }}$ Malaxa" Clinical Hospital, Bucharest, "“Euroclinic" Minimally Invasive and Reconstructive Hospital, Bucharest, ${ }^{7 " E l i a s " ~ E m e r g e n c y ~ C l i n i c a l ~ H o s p i t a l, ~ B u c h a r e s t, ~ R o m a n i a ~}$

\begin{abstract}
Aims: The aim of the study was to evaluate the diagnostic ability of sonovaginography (SVG) with ultrasound gel in patients with endometriosis. Material and methods: We conducted a multicentre prospective study, which included 193 patients with symptoms highly suggestive for endometriosis. All patients were investigated by transvaginal sonography and SVG with gel and afterwards underwent laparoscopic surgery. For each category of endometriotic lesions investigated, we calculated and compared the sensitivity, specificity, positive predictive value and negative predictive value of the imagistic investigations used. Results: In the case of endometriotic lesions of the uterosacral ligaments, SVG with gel had a sensitivity of $78.5 \%$ and a specificity of $96 \%(\mathrm{p}=\mathrm{ns})$. The lesions of the vagina and rectovaginal septum were diagnosed with a sensitivity of $79 \%$, respectively $94 \%(p=n s)$, obtaining a specificity of $99 \%$, respectively of $97 \%(p=0.007)$. The lesions of the Douglas pouch were identified with a sensitivity of $81 \%(p=0.015)$, and those of the rectosigmoid with a $94 \%$ sensitivity $(p=0.010)$. We obtained lower sensitivity (67\%) in detecting the lesions of the urinary bladder ( $p=n s)$. Conclusions: SGV with ultrasound gel represents a useful investigation tool for the evaluation of endometriotic lesions in the posterior pelvic compartment.
\end{abstract}

Keywords: sonovaginography, gel, endometriotic lesions.

\section{Introduction}

Endometriosis is a benign disease of the female reproductive tract, characterised by the pathological implantation of endometrial tissue outside the uterine cavity. The most widespread staging system of endometriosis is known as ASRM (American Society for Reproductive Medicine) clasification, which divides endometriosis implants into 4 stages depending on the intraoperative assessment of the location and extent of the lesions: Iminimal, II-mild, III-moderate, and IV-severe. The most common locations of the endometriosis implants are the

Received 26.03.2015 Accepted 19.05.2016

Med Ultrason

2016, Vol. 18, No 4, 469-474

Corresponding author: Dr. Claudia Mehedintu

"Nicolae Malaxa" Clinical Hospital, Bucharest Department of Obstetrics and Gynaecology

Vergului Street, No.12, 2nd District, Bucharest

Phone: +40722 312976

E-mail: claudiamehedintu@yahoo.com ovaries and the pelvic peritoneum. In more advanced stages of the disease, the situses of deep implantation of endometriosis are described, defined by the deep penetration of the subperitoneal tissues by the endometriotic lesions for a depth higher than $5 \mathrm{~mm}$ [1]. The locations of deep endometriosis in decreasing order of their frequency of appearance are the uterosacral ligaments (USL), Douglas pouch, rectum, sigmoid, vagina and urinary bladder [2,3]. The two main clinical manifestations of endometriosis are represented by infertility and chronic pelvic pain of variable intensity, thus affecting the quality of life. The cyclic pelvic pain, dysmenorrhoea, dyschezia, dysuria, and dyspareunia are other possible symptoms.

The preoperatively evaluation of endometriosis lesions, as precisely as possible, is important both for the appropriate counselling of the patients and for choosing the optimal therapeutic conduct. Due to the non-specific symptoms, the positive diagnosis of endometriosis bears many traps, and currently there is not an unanimously accepted standard regarding the diagnosis algorithm. The 
gynaecological clinical examination can indicate painful nodules in the Douglas pouch or in the uterosacral ligaments [4]. However, the clinical examination can be normal in many patients, having a limited capacity to quantify the extent of the endometriosis lesions $[5,6]$.

Of the available imagistic explorations, transvaginal sonography (TVS) is widely used, being an easily accessible and efficient investigation in terms of costs and time. TVS is considered the first-line imagistic investigation in the diagnosis of endometriosis [7]. The method was proven to be efficient in diagnosing the ovarian endometriomas and urinary bladder endometriosis, emphasising its lower sensitivity in the detection of the endometriotic lesions in the posterior pelvic compartment, vagina, uterosacral ligaments, and rectovaginal septum.

Taking into account the limitations of TVS, particularly in diagnosing the deep infiltrating endometriosis, Dessole et al [8] described the sonovaginography (SVG) with saline solution as a new imagistic technique. This process involves a combination of TVS with the introduction of a saline solution into the vagina that creates an acoustic window between the transvaginal probe and the surrounding structures of the vagina. Thus, an improved visualisation of the structures in the posterior pelvic compartment is achieved. This technique allows diagnosing the rectovaginal endometriosis with higher accuracy compared to TVS (sensitivity of $90.6 \%$ and a specificity of $85.7 \%$ ) [8]. The main disadvantages of this technique are the need for a second examiner, who needs to close the labia major in order to prevent the leakage of the saline solution outside the vagina, and sometimes the significant discomfort of the patient. Replacing saline solution with ultrasound gel has the advantages of eliminating the need for the second examiner and lowering the discomfort for the patient.

The main objective of our study was to evaluate the diagnostic ability of SVG with ultrasound gel as a new imagistic technique in patients with endometriosis.

\section{Material and methods}

We conducted a multi-centre prospective study between January 2011 and May 2015, within the Obstetrics-Gynaecology Clinic of "St. Pantelimon" Emergency Clinical Hospital and the Department of Gynaecology of "Euroclinic" Hospital in Bucharest. Highly suggestive symptoms for endometriosis (chronic pelvic pain, infertility, dysmenorrhoea, dyspareunia, dyschezia) were found in 193 patients, which were included in the study. The inclusion criteria were: patients of reproductive age with clinical suspicion of endometriosis and the consent for TVS and SVG with gel, as well as for the laparoscopic surgery. The exclusion criteria were represented by the existence of a malignant pathol- ogy and the presence of pregnancy. The risks and benefits of the investigations and procedures were presented to each patient and all of them signed the informed consent. Local ethics committee approval was obtained.

According to a standard protocol, each patient was investigated successively through TVS and SVG with gel by two sonographers experienced in diagnosing endometriosis (106 patients at „St. Pantelimon” Clinical Emergency Hospital Bucharest and 87 patients at "Euroclinic" Hospital Bucharest). Two ultrasound devices were used: Voluson E8 Expert (GE Healthcare) and Medison Sonoace X6, both with $7.5 \mathrm{MHz}$ transvaginal probe.

In order to perform the gel SVG, $40 \mathrm{ml}$ of ultrasound gel was introduced in the posterior vaginal fundus using a syringe. We paid full attention to completely filling the syringe with ultrasound gel in order to eliminate the air bubbles as much as possible. The syringe was also introduced as deeply as possible into the vagina, to fully fill the posterior vaginal fornix. Thus, an acoustic window was obtained, which allowed the investigation of the anterior and posterior pelvic compartment by transvaginal ultrasound (fig 1). No patient had any notable discomfort during the examination and the vagina was not necessary to be refilled with ultrasound gel in any case.

The investigation of the anterior pelvic compartment included muscular and mucous of the urinary bladder

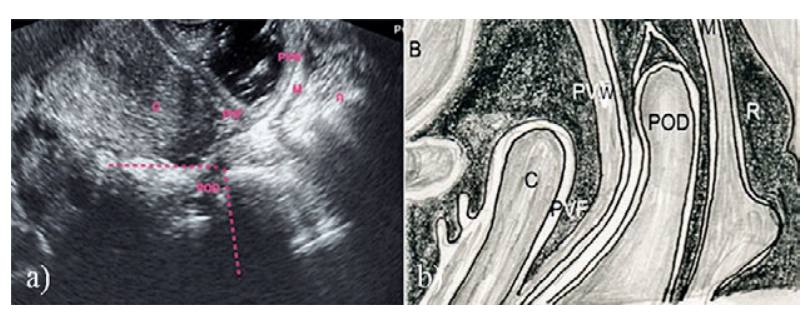

Fig 1. a) Sonovaginography using gel, sagittal plane, normal posterior compartment; b) schematic representation of normal posterior compartment using gel sonovaginography, sagittal plane. C - cervix, $\mathrm{R}$ - anterior rectum wall, POD - pouch of Douglas, PVF - posterior vaginal fornix, PVW - posterior vaginal wall, $\mathrm{M}$ - muscularis mucosae, $\mathrm{B}$ bowel.

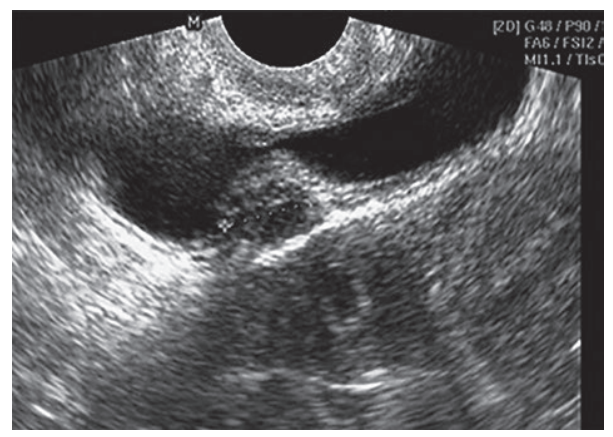

Fig 2. Transvaginal sonography image of an endometriotic nodule in the urinary bladder wall. 
walls of the, distal ureters, intramural part of the ureter, and pelvic part of the ureters $(4-6 \mathrm{~cm}$ distance above the ureterovesical junction). For this purpose the transvaginal probe was rotated $45^{\circ}$ longitudinally, thus placing it in the vesicoureteral junction; at this point, a longitudinal image of the intramural ureter was obtained. The ureter was identified by applying slight compression over the distal ureter with the transvaginal probe. The vesicouterine peritoneum was evaluated by assessing its mobility while the probe was moved on the front of the uterus. Endometriotic lesions were suspected in the presence of hypoechoic nodular structures with regular or irregular contour or linear hypoechoic thickenings in the walls of bladder or in vesicouterine space (fig 2).

Ovarian endometriomas were diagnosed through the presence of single or multiple cystic formations, with a homogenous and hypoechoic content.

In the posterior pelvic compartment the posterior wall of the vagina, posterior side of the uterus and cervix, uterosacral ligaments, rectovaginal septum, and anterior
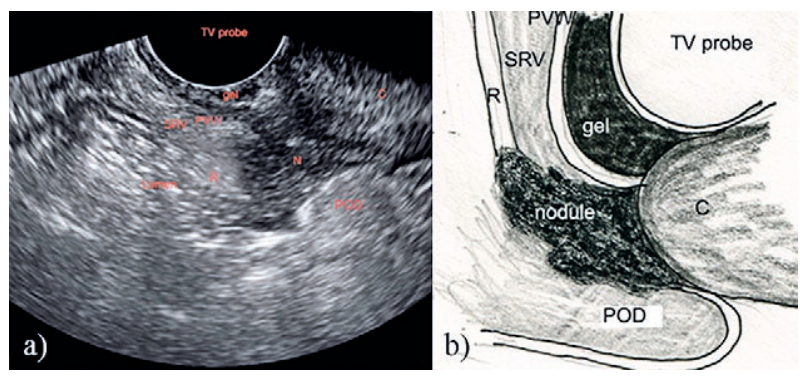

Fig 3. a) Recto-vaginal nodule viewed by gel SVG. The nodule infiltrates the rectum, obliterates the pouch of Douglas and extends caudally to the rectovaginal septum; b) Schematic representation of endometriotic nodule in the posterior compartment using gel SVG - sagittal plane. SRV - rectovaginal septum, C - cervix, $\mathrm{R}$ - anterior wall of rectum, POD - pouch of Douglas, PVW - posterior vaginal wall. walls of the recto-sigmoid were evaluated. The transvaginal probe was initially introduced in the posterior vaginal fundus. The examiner placed one hand on the lower abdominal wall, mobilising the uterus between the hand and the transvaginal probe, and a slight pressure was exerted. In this way we evaluated the mobility of the Douglas pouch, the presence of uterorectal adhesions, and the obliteration of the Douglas pouch. The posterior part of the uterus and the uterosacral ligaments were examined during withdrawal of the probe from the posterior vaginal fundus to outside. The sonographic changes of the pelvic anatomical structures suggestive for endometriosis included abnormal hypoechoic linear thickenings, asymmetry of the uterosacral ligaments and nodules/hypoechoic tumoural masses with regular or irregular contour (fig 3). The posterior vagina, the recto-sigmoid and the rectovaginal septum were investigated by moving the probe cranially and caudally on the posterior vaginal wall, corroborated with a rotating movement in different plans (fig 4). Vagina involvement was identified in the presence of thickenings and/or cystic or non-cystic nodular lesions with hypoechoic aspect situated in the posterior vaginal wall. In one case the urinary bladder lesion was identified through cystoscopy by urologist.

All patients were subjected to laparoscopic surgery. During the interventions, the surgeon evaluated all the areas investigated by sonography. All suspected lesions were excised and endometriosis was confirmed histologically. In order to remove the endometriotic lesions a partial cystectomy was performed in 2 cases of deep bladder endometriosis and colorectal "shaving" was practiced in cases of recto-sigmoid endometriosis, without requiring bowel resection.

\section{Statistical analysis}

A descriptive statistical analysis was performed according to the laparoscopy results, which were compared

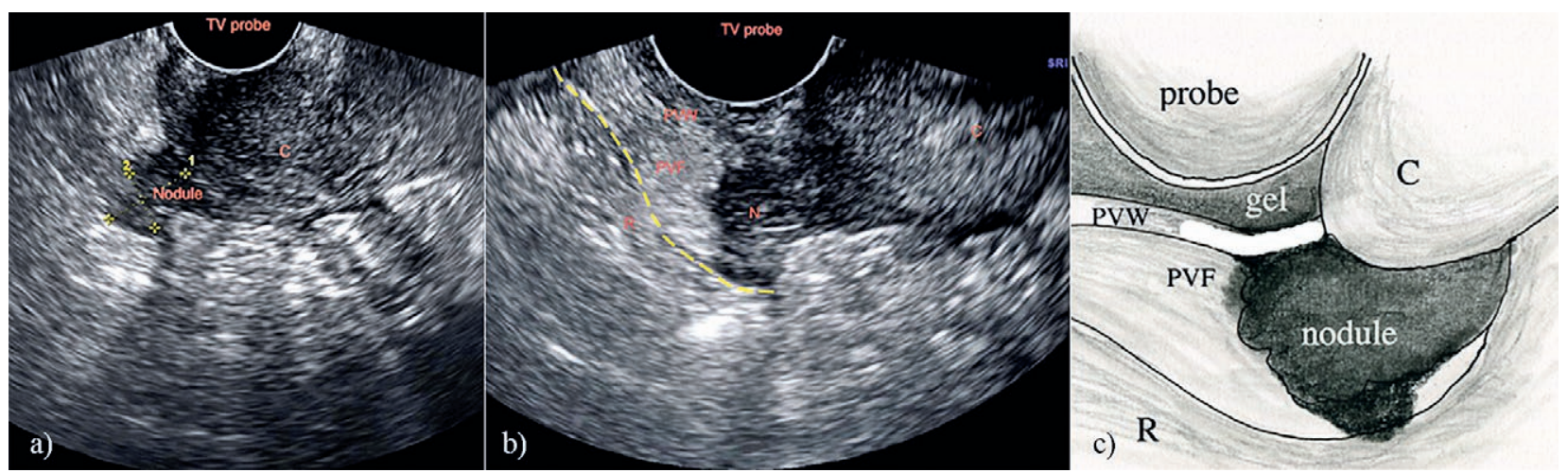

Fig 4. a) Recto-vaginal endometriosis nodule viewed by standard transvaginal ultrasound; b) Recto-vaginal endometriotic nodule viewed by gel SVG. The nodule infiltrates the rectum, without infiltrating caudally the rectovaginal septum; c) Schematic representation of endometriotic nodule in the posterior compartment using gel SVG - sagittal plane. $\mathrm{C}$ - cervix, $\mathrm{R}$ - anterior wall of the rectum, $\mathrm{PVF}$ - posterior vaginal fornix, PVW, posterior vaginal wall, $\mathrm{N}$ - nodule. 
to the ultrasound findings. Patient age is presented using the mean and SD (standard deviation); binary variables are presented as number and percentage (\%), with P-values calculated using Fisher's exact test. $p<0.05$ was considered statistically significant. Obtained data was analysed by comparing some statistical indicators specific to each individual implantation situs: sensitivity, specificity, positive and negative predictive values. The data were processed with the SPSS software, version 15.0 (IBM Corporation).

\section{Results}

The mean age $\pm \mathrm{SD}$ of the 193 patients was $32 \pm 4.3$ years. The clinical signs and symptoms encountered within the studied lot were represented by: chronic pelvic pain in 183 patients (94.8\%), dysmenorrhoea in 193 patients $(100 \%)$, dyspareunia in 147 patients $(76.1 \%)$, dyschezia in 48 patients $(24.8 \%)$, and infertility in 120 patients $(62.1 \%)$.

After the laparoscopic intervention, 145 (75.12\%) of the patients were diagnosed with moderate forms of endometriosis, while the remaining 48 (24.88\%) had severe endometriosis, according to ASRM classification. In 45 patients $(23.31 \%)$ ovarian endometriomas and endometriotic lesions in the anterior and posterior pelvic compartment were identified. In 44 cases $(22.79 \%)$ ovarian endometriomas and endometriotic lesions in the poste- rior compartment were found, while the remaining 104 patients $(53.9 \%)$ only had ovarian endometriomas on ultrasound examination. In the 193 patients confirmed with moderate and severe endometriosis, 204 endometriotic lesions were identified after laparoscopy, some patients having more than 1 lesion. The distribution of these lesions is shown in Table I. Of the 96 intraoperative identified lesions in the posterior compartment (uterosacral ligaments, vagina, rectovaginal septum, pouch of Douglas, recto-sigmoid), deep endometriosis was identified in 38 patients $(19.6 \%)$.

Of the 18 lesions of the urinary bladder, 4 lesions involved the full thickness of the bladder wall until the mucosal lining, and in one certain case the lesion was identified through cystoscopy by an urologist. Of the 4 cases of deep

Table I. Endometriotic lesions classified based on a specific site in ther anterior and posterior pelvic compartment (some patients had more than 1 lesion)

\begin{tabular}{ll}
\hline Location of endometriotic lesions & n \\
\hline Ovary & 90 \\
Urinary bladder & 18 \\
Uterosacral ligaments & 10 \\
Vagina & 12 \\
Rectovaginal septum & 12 \\
Pouch of Douglas & 48 \\
Recto-sigmoid & 14 \\
Total & $\mathbf{2 0 4}$ \\
\hline
\end{tabular}

Table II. TVS and gel SVG diagnostic accuracy of the endometriotic pelvic lesions

\begin{tabular}{|c|c|c|c|c|c|}
\hline Endometriotic lesion & $\mathbf{U S}$ & Se (\%) & Sp (\%) & PPV (\%) & NPV (\%) \\
\hline \multirow[t]{3}{*}{ Ovaries } & TVS & $94.8 \%$ & $95.6 \%$ & $92.5 \%$ & $94 \%$ \\
\hline & SVG with gel & $95.5 \%$ & $97 \%$ & $92.6 \%$ & $93 \%$ \\
\hline & $\mathrm{p}$ & 0.733 & 0.471 & 0.480 & 0.722 \\
\hline \multirow[t]{3}{*}{ Urinary bladder } & TVS & $65.5 \%$ & $98.6 \%$ & $66.6 \%$ & $98.5 \%$ \\
\hline & SVG with gel & $67 \%$ & $97 \%$ & $98 \%$ & $98 \%$ \\
\hline & $\mathrm{p}$ & 0.721 & 0.252 & 0.279 & 0.745 \\
\hline Uterosacral & TVS & $69.7 \%$ & $95 \%$ & $84.2 \%$ & $87 \%$ \\
\hline \multirow[t]{2}{*}{ Ligaments } & SVG with gel & $78.5 \%$ & $96 \%$ & $89 \%$ & $91 \%$ \\
\hline & $\mathrm{P}$ & 0.614 & 0.609 & 0.599 & 0.648 \\
\hline \multirow[t]{3}{*}{ Vagina } & TVS & $58.5 \%$ & $99 \%$ & $94.3 \%$ & $95.4 \%$ \\
\hline & SVG with gel & $79 \%$ & $99 \%$ & $92 \%$ & $96 \%$ \\
\hline & $\mathrm{p}$ & 0.398 & 1.000 & 0.885 & 0.484 \\
\hline Rectovaginal & TVS & $64.8 \%$ & $99.4 \%$ & $81.5 \%$ & $97.8 \%$ \\
\hline \multirow[t]{2}{*}{ Septum } & SVG with gel & $94 \%$ & $97 \%$ & $82 \%$ & $93.5 \%$ \\
\hline & $\mathrm{p}$ & 0.132 & 0.007 & 0.018 & 0.013 \\
\hline \multirow[t]{3}{*}{ Pouch of Douglas } & TVS & $81.5 \%$ & $99 \%$ & $95 \%$ & $97 \%$ \\
\hline & SVG with gel & $81 \%$ & $98 \%$ & $91 \%$ & $97 \%$ \\
\hline & $\mathrm{p}$ & 0.015 & 0.032 & 0.013 & 1.000 \\
\hline \multirow[t]{3}{*}{ Recto-sigmoid } & TVS & $91 \%$ & $97 \%$ & $95 \%$ & $96 \%$ \\
\hline & SVG with gel & $94 \%$ & $95.5 \%$ & $91 \%$ & $97.2 \%$ \\
\hline & $\mathrm{p}$ & 0.010 & 0.049 & 0.013 & 0.035 \\
\hline
\end{tabular}

US - ultrasonographic investigation, Se - sensitivity, Sp - specificity, PPV - positive predictive value, NPV - negative predictive value, TVS - transvaginal sonography, SGV - sonovaginography. 
Table III. The diagnostic capacity of TVS and SVG with gel, depending on the location in the anterior and posterior pelvic compartment of the endometriotic lesions

\begin{tabular}{llllll}
\hline Pelvic compartment & US & Se (\%) & Sp (\%) & PPV (\%) & NPV (\%) \\
\hline Anterior & TVS & $80.1 \%$ & $97.1 \%$ & $79.5 \%$ & $96.2 \%$ \\
& SVG with gel & $81.2 \%$ & $97 \%$ & $95.3 \%$ & $95.5 \%$ \\
\multirow{3}{*}{ Posterior } & p & 0.433 & 0.326 & 0.234 & 0.146 \\
& TVS & $73.1 \%$ & $97.8 \%$ & $90 \%$ & $94.6 \%$ \\
& SVG with gel & $85.3 \%$ & $97.1 \%$ & $89 \%$ & $94.9 \%$ \\
& p & 0.051 & $\mathbf{0 . 0 2 6}$ & $\mathbf{0 . 0 0 3}$ & $\mathbf{0 . 0 1 5}$ \\
\hline
\end{tabular}

US - ultrasonographic investigation, Se - sensitivity, $\mathrm{Sp}$ - specificity, PPV - positive predictive value, NPV - negative predictive value, TVS - transvaginal sonography, SGV - sonovaginography.

bladder endometriosis, partial cystectomy was performed in 2 cases $(1.03 \%)$. The remaining 14 bladder lesions were superficial. From the 38 patients with deep endometriosis, through the speculum examination the lesion was highlighted on the posterior vaginal mucosa in 3 cases $(1.55 \%)$.

Table II shows the capacity of the SVG with gel to detect various types of endometriotic lesions.

Table III analyses the capacity to diagnose the endometriotic lesions through TVS and SVG with gel, depending on the location in the anterior and posterior pelvic compartment, evidencing a superior capacity of SVG with gel to detect the lesions in the posterior pelvic compartment.

\section{Discussions}

Our study showed that SVG with gel is a good method in evaluating patient with deep endometriosis. By examining the two pelvic compartments with this method a correct staging of the endometriotic lesions was possible, which enabled the proper counselling of patients, as well as proper planning of surgical intervention. By analysing the data achieved, we observed that TVS and SVG with gel have some sensitivity without a significant statistical difference $(80.1 \%$ respectively $81.2 \%)$ in diagnosing the endometriosis of the anterior pelvic compartment. According to the same results, SVG with gel proved to be more efficient in detecting the lesions of the posterior pelvic compartment, with a sensitivity of $85.3 \%$ compared to $73.1 \%$ obtained by means of TVS $(\mathrm{p}=0.051)$.

The lower capacity in the diagnosis of lesions of the urinary bladder can be explained by the probability that some of the patients were examined with a semi-full or even empty urinary bladder or lesions were too small to be detected by sonography. From this point of view, our results are consistent with the results of Savelli et al [9] which reported a sensitivity of $44 \%$ of gel SVG in the diagnosis of vesical endometriosis.

Gel SVG allows the identification of endometriotic pelvic nodules and of the structures that are secondary infiltrated (rectovaginal septum, rectal wall, posterior vaginal wall) and the depth of the invasion. That is why gel SVG should be performed complementary to TVS if deep endometriosis is suspected in order to more accurately assess the extent of the lesions of the rectovaginal septum and the anterior rectal wall. In addition to the benefits of diagnostic accuracy compared to TVS in assessing the posterior pelvic compartment, gel SVG allows a more easily identification of the uterosacral ligaments, rectovaginal septum, and posterior vaginal wall.

Taking into account the detection of the lesions of uterosacral ligaments through SVG with gel, we obtained a slightly lower sensitivity compared to the study conducted by Leon et al [10], who reported a sensitivity of $84 \%$, but the same specificity of $96 \%$.

One of the purposes of the study was to determine whether SVG with gel is useful in diagnosing the endometriotic lesions in the vagina and rectovaginal septum. The results obtained provide an encouraging perspective in this regard, compared to the results obtained by transvaginal ultrasound by Hudelist et al [11] who obtained a sensitivity of $64 \%$ for vaginal lesions, respectively $78 \%$ for the lesions of the rectovaginal septum. Reid et al [12] obtained a sensitivity of $83 \%$ and a specificity of $97 \%$ in the detection of the obstruction of the pouch of Douglas, the results being similar to ours. The diagnosis of ovarian endometriomas through SVG with gel had a sensitivity of $95.5 \%$, smaller than the one obtained by using the TVS by Hudelist et al [13], which communicated a sensitivity of $98 \%$ in the detection of these lesions. These results determined us to consider TVS to be superior to SVG in diagnosing ovarian endometriosis.

Our study had also some limitations. Some endometriotic lesions were too small to be detected by sonography and could have been missed. Another limitation was that TVS and SVG were performed by the same operator, which presents a potential bias for the SVG findings. Another source of bias was that the surgeon was aware of the sonographic findings before surgery. 
We consider this study a starting point for the evaluation of the gel SVG as a reliable technique in the diagnosis of posterior pelvic compartment endometriosis. Studies with larger populations and sonographers of different skill levels are required in order to confirm the diagnostic accuracy of gel SVG.

\section{Conclusions}

SVG with gel is a simple investigation successfully used in diagnosing the endometriotic lesions of the posterior pelvic compartment. We consider that, compared to TVS, SVG with gel provides an improved evaluation of the posterior pelvic compartment, particularly of the posterior vaginal fornix, rectovaginal septum and cervix. The investigation allows the evaluation of the lesions of the recto-sigmoid and rectovaginal septum, providing a true advantage in guiding the operating strategy and possibly involving in the team a surgeon experienced in colorectal surgery.

\section{Conflict of interest: none}

\section{References}

1. Koninckx PR, Meuleman C, Demeyere S, Lesaffre E, Cornillie FJ. Suggestive evidence that pelvic endometriosis is a progressive disease, whereas deeply infiltrating endometriosis is associated with pelvic pain. Fertil Steril 1991;55:759-765.

2. Jenkins S, Olive DL, Haney AF. Endometriosis: pathogenetic implications of the anatomic distribution. Obstet Gynecol 1986;67:335-338.

3. Cornillie FJ, Oosterlynck D, Lauweryns JM, Koninckx PR. Deeply infiltrating pelvic endometriosis: histology and clinical significance. Fertil Steril 1990;53:978-983.
4. Cheewadhanaraks S, Peeyananjarassri K, Dhanaworavibul $\mathrm{K}$, Liabsuetrakul T. Positive predictive value of clinical diagnosis of endometriosis. J Med Assoc Thai 2004;87:740744.

5. Koninckx PR, Martin D. Treatment of deeply infiltrating endometriosis. Curr Opin Obstet Gynecol 1994;6:231-241.

6. Chapron C, Dubuisson JB, Pansini V, et al. Routine clinical examination is not sufficient for diagnosing and locating deeply infiltrating endometriosis. J Am Assoc Gynecol Laparosc 2002;9:115-119.

7. Bazot M, Malzy P, Cortez A, Roseau G, Amouyal P, Darai E. Accuracy of transvaginal sonography and rectal endoscopic sonography in the diagnosis of deep infiltrating endometriosis. Ultrasound Obstet Gynecol 2007;30:994-1001.

8. Dessole S, Farina M, Rubattu G, Cosmi E, Ambrosini G, Nardelli GB. Sonovaginography is a new technique for assessing rectovaginal endometriosis. Fertil Steril 2003;79:1023-1027.

9. Savelli L, Manuzzi P, Pollastri M, Mabrouk M, Seracchioli R, Venturoli S. Diagnostic accuracy and potential limitations of transvaginal sonography for bladder endometriosis. Ultrasound Obstet Gynecol 2009;34:595-600.

10. León M, Vaccaro H, Alcázar JL, et al. Extended transvaginal sonography in deep infiltrating endometriosis: use of bowel preparation and an acoustic window with intravaginal gel: preliminary results. J Ultrasound Med 2014;33:315-321.

11. Hudelist G, Ballard K, English J, et al. Transvaginal sonography vs. clinical examination in the preoperative diagnosis of deep infiltrating endometriosis. Ultrasound Obstet Gynecol 2011;37:480-487.

12. Reid S, Lu C, Casikar I, et al. Prediction of pouch of Douglas obliteration in women with suspected endometriosis using a new real-time dynamic transvaginal ultrasound technique: the sliding sign. Ultrasound Obstet Gynecol 2013;41:685-691.

13. Hudelist G, Oberwinkler KH, Singer CF, et al. Combination of transvaginal sonography and clinical examination for preoperative diagnosis of pelvic endometriosis. Hum Reprod 2009;24:1018-1024. 